\title{
SYLOW INTERSECTIONS, DOUBLE COSETS, AND 2-BLOCKS
}

\author{
J. Murray* \\ Mathematics Department, \\ University College Dublin, \\ Belfield Dublin 4, \\ Ireland.
}

\section{Notation And statement of Results}

Throughout $G$ will be a finite group and $F$ will be a finite field of characteristic $p>0$, although we are mainly interested in the case $p=2$. For convenience we assume that $F$ is a splitting field for all subgroups of $G$. We let $\mathbb{Z}_{(p)}$ denote the localization of the integers $\mathbb{Z}$ at the prime ideal $p \mathbb{Z}$. If $x \in \mathbb{Z}_{(p)}$, then $x^{*}$ will denote its image modulo the unique maximal ideal of $\mathbb{Z}_{(p)}$. We regard $x^{*}$ as lying in the prime field $\mathrm{GF}(p)$ of $F$.

The elements of $G$ may be identified with the members of a distinguished basis of the group algebra $F G$. Thus each $x \in F G$ is of the form $x=$ $\sum_{g \in G} \beta(x, g) g$, where $\beta(x, g) \in F$, for $g \in G$. We define the element $x^{o}$ of $F G$ as $x^{o}:=\sum_{g \in G} \beta\left(x, g^{-1}\right) g$. The map $x \rightarrow x^{o}$ is an anti-isomorphism of $F G$, and its restriction to the centre $Z$ of $F G$ is an involutary isomorphism. We use $\mathcal{K}^{+}$to denote the sum of the elements in a $G$-conjugacy class $\mathcal{K}$ in $F G$.

1991 Mathematics Subject Classification. 20C20.

*The author was supported by an Enterprise Ireland research grant while writing this paper. 
The set of all such class sums forms an $F$-basis for $Z$. If $x \in Z$ and $g \in \mathcal{K}$, we will use $\beta\left(x, \mathcal{K}^{+}\right)$in place of $\beta(x, g)$.

By a $p$-block $B$ of $G$ we mean a direct $F$-algebra summands of $F G$. Associated with $B$ there is a primitive idempotent $e \in Z$, and an $F$-epimorphism $\omega: Z \rightarrow F$. We indicate these associations by $B \leftrightarrow e \leftrightarrow \omega$, and call $e$ the block idempotent, and $\omega$ the central character, of $B$. Set $B^{o}:=\left\{x \in F G \mid x^{o} \in B\right\}$ and $\omega^{o}(x):=\omega\left(x^{o}\right)$. Then $B^{o} \leftrightarrow e^{o} \leftrightarrow \omega^{o}$ is a $p$-block of $G$. We say that $B$ is a real block of $G$ if $B=B^{o}$.

R. Brauer showed how to associate with $B$ a $G$-conjugation family of $p$ groups, which he called the defect groups of $B$. Let $D$ be a defect group of $B$. Then $D$ is not arbitrarily embedded in $G$. For instance Brauer proved that $D$ is the largest normal $p$-subgroup of its normalizer $\mathbf{N}(D)$. J. A. Green [5] showed that there exists $g \in G$ and a Sylow $p$-subgroup $S$ of $G$, such that $S \cap S^{g}=D$, and M. F. O'Reilly [9] showed that $g$ could be chosen to be $p$-regular with defect group $D$. Here a defect group of $g$ means a Sylow $p$-subgroup of the centralizer $\mathbf{C}(g)$ of $g$ in $G$.

Let $e_{D}$ denote the sum of the block idempotents associated with the $p$-blocks of $G$ which have defect group $D$, and let $\mathcal{S} y l$ denote the collection of Sylow $p$-subgroups of $G$. We prove the following partial converse to these results:

Theorem 2.9. Let $p=2$ and let $g \in G$ be 2-regular with defect group $D$. Then $\beta\left(e_{D}, g\right)=\left|\left\{P \in \mathcal{S} y l \mid P \cap P^{g}=D, P g P=P g^{-1} P\right\}\right| 1_{F}$.

Now $G$ has a 2-block with defect group $D$ if and only if $\beta\left(e_{D}, g\right) \neq 0$ for some 2-regular element $g$ with defect group $D$. So 2.9 furnishes a necessary and sufficient condition for $G$ to have a 2-block with defect group $D$. 
If $g \in G$, set $\mathbf{C}^{*}(g):=\left\{x \in G \mid g^{x} \in\left\{g, g^{-1}\right\}\right\}$. We call the Sylow 2subgroups of $\mathbf{C}^{*}(g)$ the extended defect groups of $g$. Let $\mathcal{K}$ be the conjugacy class of $G$. The extended defect groups of the elements of $\mathcal{K}$ form a single $G$-orbit. which we call the extended defect groups of $\mathcal{K}$. If $E$ is an extended defect group of $g$, then $D:=\mathbf{C}_{E}(g)$ is a group of $g$ which is contained in $E$, and $|E: D| \leq 2$. We call $(D, E)$ a defect pair for $g$. The defect pairs of the elements of $\mathcal{K}$ form a single $G$-orbit. We call $g$ a real element if it is $G$-conjugate to $g^{-1}$. Theorem 2.9 can be refined for real elements as follows:

Theorem 3.1. Let $p=2$ and let $g \in G$ be real and 2-regular with defect pair $(D, E)$. Then $\beta\left(e_{D}, g\right)=\left|\left\{P \in \mathcal{S} y l \mid P \cap P^{g}=D, E \leq P\right\}\right| 1_{F}$.

We use this theorem to give an alternative proof of Theorem 4.8 of [4] and also to provide a self-contained treatment of some results of M. Herzog.

Let $\mathcal{K}$ be a conjugacy class of $G$. We call $\mathcal{K}$ a real class if it coincides with its inverse class $\mathcal{K}^{o}:=\left\{g \in G \mid g^{-1} \in \mathcal{K}\right\}$. We call a real class $\mathcal{K}$ properly real if $g^{2} \neq 1$ for $g \in \mathcal{K}$.

Suppose that $\mathcal{K}$ and $\mathcal{L}$ are conjugacy classes of $G$. We write

$$
\mathcal{K} \leq \mathcal{L}
$$

if each defect group of $\mathcal{K}$ is contained in some defect group of $\mathcal{L}$. Suppose in addition that $\mathcal{K}$ is properly real and that $\mathcal{L}$ is real. We write

$$
\mathcal{K} \preceq \mathcal{L}
$$

if for each defect pair $(D, E)$ of $\mathcal{K}$, there exists $l \in \mathcal{L}$ such that $D \leq \mathbf{C}(l)$ and $E \leq \mathbf{C}^{*}(l)$, but $E \not \mathbf{C}(l)$ if $l^{2} \neq 1$.

Let $B \leftrightarrow e \leftrightarrow \omega$ be a 2-block of $G$. It is well-known that there exists a 2 -regular class $\mathcal{L}$ of $G$ such that $\beta\left(e, \mathcal{L}^{+}\right) \neq 0$ and $\omega\left(\mathcal{L}^{+}\right) \neq 0$. Any such $\mathcal{L}$ is 
called a defect class for $B$. The min-max theorem $[7,15.31]$ states that

$$
\begin{aligned}
\omega\left(\mathcal{K}^{+}\right) \neq 0 & \Longrightarrow \mathcal{L} \leq \mathcal{K}, \quad \text { for each class } \mathcal{K} \text { of } G . \\
\beta\left(e, \mathcal{K}^{+}\right) \neq 0 & \Longrightarrow \mathcal{K} \leq \mathcal{L},
\end{aligned}
$$

Suppose that $B$ is a real 2-block of $G$. R. Gow showed in [3] that $B$ has a defect class $\mathcal{K}$ which is real. Let $(D, E)$ be a defect pair for $\mathcal{K}$. Gow proved that the extended defect groups of the real defect classes of $B$ are $G$-conjugate to $E$. For this reason he referred to the $G$-conjugates of $E$ as the extended defect groups of $B$. We call $(D, E)$ a defect pair for $B$. Theorem 2.1 of [3] can be extended in the following way:

Theorem 4.3 (Min-Max for Real 2-Blocks). Let $B \leftrightarrow e \leftrightarrow \omega$ be a real nonprincipal 2-block of $G$ and let $\mathcal{L}$ be a real defect class of $B$. Then

$$
\begin{aligned}
\omega\left(\mathcal{K}^{+}\right) \neq 0 & \Longrightarrow \mathcal{L} \preceq \mathcal{K}, \quad \text { for each real class } \mathcal{K} \text { of } G . \\
\beta\left(e, \mathcal{K}^{+}\right) \neq 0 & \Longrightarrow \mathcal{K} \preceq \mathcal{L},
\end{aligned}
$$

Throughout the paper $S$ will be a fixed Sylow 2-subgroup of $G$. Let $D \leq E$ be subgroups of $S$ with $|E: D|=2$. Let $S \backslash G / S$ denote a set of representatives for the double cosets of $S$ in $G$. If $x, y \in G$ lie in the same $(S, S)$-double coset, then the groups $S \cap S^{x}$ and $S \cap S^{y}$ are $S$-conjugate. We say that $S g S$ is a self-dual double coset if $S g S=S g^{-1} S$. Lemma 5.1 furnishes a 2-subgroup $\left(S \cap S^{g}\right)^{*}$ of $G$ which contains $S \cap S^{g}$ as a subgroup of index 2, whenever $S g S$ is self-dual and distinct from $S$. Moreover, if $x, y \in S g S$, then $\left(S \cap S^{x}\right)^{*}$ and $\left(S \cap S^{y}\right)^{*}$ are conjugate in $S$.

If $H$ and $K$ are subgroups of $G$, we write $H={ }_{G} K$ if some $G$-conjugate of $H$ equals $K$, and we write $H \leq_{G} K$ if some $G$-conjugate of $H$ is contained in $K$. We let $\sum_{x}^{D}$ denote a sum which ranges over those elements $x$ of $S \backslash G / S$ 
for which $S \cap S^{x}={ }_{G} D$, and let $\sum_{x \equiv x^{-1}}^{D}$ denote the restriction of this sum to the self-dual double cosets.

Suppose that $\left\{\mathcal{K}_{1}, \ldots, \mathcal{K}_{v}\right\}$ is a complete list of the real 2-regular classes of $G$ which have defect pair $(D, E)$. We call a self-dual double coset $S g S$ a $(D, E)$-double coset if there exists $x \in S g$ which simultaneously satisfies:

(1) $x \in \mathcal{K}_{1} \cup \cdots \cup \mathcal{K}_{v}$;

(2) $\left(S \cap S^{g},\left(S \cap S^{g}\right)^{*}\right)$ is a defect pair for $x$.

Let $x_{1}, \ldots, x_{w}$ be a (possibly empty) set of representatives for the $(D, E)$ double cosets of $S$. Suppose that $w \neq 0$. We define an $v \times w$ integer matrix $N$ by setting the $i, j$-th entry of $N$ to be the number $N_{i j}$ of $y_{i}$ in $\mathcal{K}_{i} \cap S x_{j}$ such that $\left(S \cap S^{x_{j}},\left(S \cap S^{x_{j}}\right)^{*}\right)$ is a defect pair for $y_{i}$. When $p=2$, Theorem A of [11] can be refined as follows:

Theorem 5.2. The number of real 2-blocks of $G$ which have defect pair $(D, E)$ is zero, if $w=0$, and is the 2 -rank of the matrix $N \cdot N^{T}$, if $w \neq 0$.

\section{Sylow intersections AND 2-BLOCKS}

Our starting point is Proposition 3.1 of [4]:

Lemma 2.1. Let $B \leftrightarrow e \leftrightarrow \omega$ be a p-block of $G$ which has defect group $D$ and let $\mathcal{K}$ be a p-regular class of $G$ which has defect group $D$. Then

$$
\beta\left(e, \mathcal{K}^{+}\right)=\left(\frac{\operatorname{dim}(B)}{|G||\mathcal{K}|}\right)^{*} \omega\left(\mathcal{K}^{o+}\right) .
$$

Let $J Z$ denote the Jacobson radical of $Z$ and let $E Z$ denote the $F$-span of the idempotents in $Z$. Then $J Z$ is an ideal of $Z, E Z$ is a direct sum of copies of $F$ (as an $F$-algebra) and $Z=J Z \bigoplus E Z$ as $F$-algebras. The Robinson map is the natural $F$-algebra projection $\epsilon: Z \rightarrow E Z$ with respect to this 
decomposition. Let $z \in Z$ and let $n$ be a positive integer such that $g^{p^{n}}=1_{G}$, for each $p$-element $g$ of $G$, and $\lambda^{p^{n}}=\lambda$, for each $\lambda \in F$. Then $\epsilon(z)=z^{p^{n}}$. We also have

$$
\epsilon(z)=\sum_{B} \omega(z) e
$$

where $B \leftrightarrow e \leftrightarrow \omega$ ranges over the $p$-blocks of $G$. See [12] for further details.

For the rest of the paper we take $p=2$ and $\operatorname{Char}(F)=2$. Recall that $e_{D}$ denotes the sum of the block idempotents in $Z$ which have defect group $D$. We combine Lemma 2.1 and (2.2) as follows:

Corollary 2.3. Let $\mathcal{K}$ be a 2-regular class of $G$ which has defect group $D$. Then $\beta\left(e_{D}, \mathcal{K}^{+}\right)=\beta\left(\epsilon\left(\mathcal{K}^{o+}\right), \mathcal{K}^{+}\right)$.

Proof. It follows from (1.2) and (2.2) that

$$
\beta\left(\epsilon\left(\mathcal{K}^{o+}\right), \mathcal{K}^{+}\right)=\sum_{B} \omega\left(\mathcal{K}^{o+}\right) \beta\left(e, \mathcal{K}^{+}\right),
$$

where $B \leftrightarrow e \leftrightarrow \omega$ ranges over the 2-blocks of $G$ which have defect group $D$. Also $(\operatorname{dim}(B) /|G||\mathcal{K}|)^{*}=1_{F}$, for each such $B$. Thus

$$
\begin{array}{rlrl}
\beta\left(\epsilon\left(\mathcal{K}^{o+}\right), \mathcal{K}^{+}\right) & =\sum \beta\left(e, \mathcal{K}^{+}\right)^{2}, & \text { using Lemma } 2.1 \\
& =\left(\sum \beta\left(e, \mathcal{K}^{+}\right)\right)^{2}, \quad \text { as } F \text { has characteristic } 2 \\
& =\beta\left(e_{D}, \mathcal{K}^{+}\right)^{2} & \\
& =\beta\left(e_{D}, \mathcal{K}^{+}\right), \quad \text { as } \beta\left(e_{D}, \mathcal{K}^{+}\right) \in \mathrm{GF}(2) .
\end{array}
$$

If $\mathcal{K}$ and $\mathcal{L}$ are 2-regular classes which have defect group $D$ then

$$
\beta\left(\epsilon\left(\mathcal{K}^{+}\right), \mathcal{L}^{+}\right)=\sum_{x}^{D}|\mathcal{K} \cap S x||\mathcal{L} \cap S x| 1_{F},
$$


using 1.3.3 and 1.3.4 of [12]. This allows us to prove:

Proposition 2.5. Let $\mathcal{K}$ be a 2-regular class with defect group $D$. Then $\beta\left(e_{D}, \mathcal{K}^{+}\right)=\sum_{x \equiv x^{-1}}^{D}|\mathcal{K} \cap S x| 1_{F}$.

Proof. By Corollary 2.3 and (2.4) we have

$$
\beta\left(e_{D}, \mathcal{K}^{+}\right)=\sum_{x}^{D}\left|\mathcal{K}^{o} \cap S x\right||\mathcal{K} \cap S x| 1_{F} .
$$

Let $x \in G$. The map $s x \leftrightarrow(s x)^{-s}$, for $s \in S$, establishes a bijection between the sets $\mathcal{K} \cap S x$ and $\mathcal{K}^{o} \cap S x^{-1}$. So

$$
|\mathcal{K} \cap S x|=\left|\mathcal{K}^{o} \cap S x^{-1}\right|
$$

Suppose $S x S \neq S x^{-1} S$. Then the contribution of these cosets to (2.6) is $\left|\mathcal{K}^{o} \cap S x\right||\mathcal{K} \cap S x| 1_{F}+\left|\mathcal{K}^{o} \cap S x^{-1}\right|\left|\mathcal{K} \cap S x^{-1}\right| 1_{F}=2 \cdot\left|\mathcal{K}^{o} \cap S x\right||\mathcal{K} \cap S x| 1_{F}=0_{F}$.

It follows that

$$
\beta\left(e_{D}, \mathcal{K}^{+}\right)=\sum_{x \equiv x^{-1}}^{D}\left|\mathcal{K}^{o} \cap S x\right||\mathcal{K} \cap S x| 1_{F} .
$$

Suppose that $S x S=S x^{-1} S$. Then

$$
\begin{aligned}
\left|\mathcal{K}^{o} \cap S x\right| & =\left|\mathcal{K}^{o} \cap S x^{-1}\right|, \\
& =|\mathcal{K} \cap S x|, \quad \text { as } S x^{-1} \text { and } S x \text { are } S \text {-conjugate }
\end{aligned}
$$

We conclude from (2.8) and the fact that the prime field of $F$ is GF(2) that

$$
\beta\left(e_{D}, \mathcal{K}^{+}\right)=\sum_{x \equiv x^{-1}}^{D}|\mathcal{K} \cap S x|^{2} 1_{F}=\sum_{x \equiv x^{-1}}^{D}|\mathcal{K} \cap S x| 1_{F} .
$$


Proof of Theorem 2.9. Recall that $g$ is a 2-regular element of $G$ with defect group $D$. Let $\mathcal{K}$ be the class of $G$ which contains $g$. We shall compute $\left|\left\{(k, P) \in \mathcal{K} \times \mathcal{S} y l \mid P k P=P k^{-1} P, P \cap P^{k}={ }_{G} D\right\}\right|$ in two different ways. On the one hand it equals $|\mathcal{K}||\mu(g)|$, where

$$
\mu(g):=\left\{P \in \mathcal{S} y l \mid P g P=P g^{-1} P, P \cap P^{g}={ }_{G} D\right\}
$$

On the other hand it equals $|\mathcal{S} y l| \sum_{x \equiv x^{-1}}^{D}|\mathcal{K} \cap S x S|$. The double coset $S x S$ is a union of $\left|S: S \cap S^{x}\right|$ right cosets of $S$, and each of these is $S$-conjugate to $S x$. It follows that $|\mathcal{K} \cap S x S|=\left|S: S \cap S^{x}\right||\mathcal{K} \cap S x|$. Also $\left|S: S \cap S^{x}\right|=|S: D|$, whenever $S \cap S^{x}$ is $G$-conjugate to $D$. But $|\mathcal{S} y l|$ is odd, by Sylow's Theorem. Thus

$$
\begin{aligned}
|\mu(g)| 1_{F} & =\frac{|S: D|}{|\mathcal{K}|} \sum_{x \equiv x^{-1}}^{D}|\mathcal{K} \cap S x| 1_{F} \\
& =\sum_{x \equiv x^{-1}}^{D}|\mathcal{K} \cap S x| 1_{F}, \quad \text { as } \mathcal{K} \text { has defect group } D \\
& =\beta\left(e_{D}, g\right), \quad \text { by Proposition } 2.5 .
\end{aligned}
$$

We claim that $D$ acts by conjugation on $\mu(g)$. For, suppose that $P \in \mu(g)$ and $d \in D$. Then $d g=g d$. So $P^{d} \cap P^{d g}=\left(P \cap P^{g}\right)^{d}={ }_{G} D$, and $P^{d} g P^{d}=$ $(P g P)^{d}=\left(P g^{-1} P\right)^{d}=P^{d} g^{-1} P^{d}$. Thus $P^{d} \in \mu(g)$, which proves our claim.

Each $D$-orbit in $\mu(g)$ has 2-power order, and $P$ is stabilized by $D$ if and only if $D \leq P$. But $D \leq P$ implies that $D \leq P \cap P^{g}$. Since $P \cap P^{g}={ }_{G} D$, it follows that $P$ is stabilized by $D$ if and only if $P \cap P^{g}=D$. We conclude that

$$
|\mu(g)| \equiv\left|\left\{P \in \mathcal{S} y l \mid P \cap P^{g}=D, P g P=P g^{-1} P\right\}\right|(\bmod 2),
$$

from which the theorem follows. 
Theorem 2.9 has no obvious analogue for odd primes. For instance, if $P$ is a Sylow 3-subgroup of $\operatorname{PSL}_{3}(2)$ and $g$ is an element of order 4, the set $\left\{P \in \mathcal{S} y l \mid P \cap P^{g}=\{1\}, P g P=P g^{-1} P\right\}$ has cardinality 4. However, $g$ has 3-defect zero and appears with zero multiplicity in the sum of the 3-block idempotents of defect zero.

We indicate how our methods may be used to sharpen Corollary 2 of [11]:

Theorem 2.10. Let $g$ be a 2-regular element of $G$ which has defect group $D$. Suppose that $P \cap P^{g}=D$, for each Sylow 2-subgroup $P$ of $G$ which contains $D$. Then $g$ lies in a defect class of some real 2-block of $G$. In particular, $G$ has a real 2-block with defect group $D$.

Proof. Let $r_{D}$ denote the sum of the real 2-block idempotents of $G$ which have defect group $D$, and let $\mathcal{K}$ be the conjugacy class of $G$ which contains $g$. We can show that

$$
\beta\left(r_{D}, \mathcal{K}^{+}\right)=\beta\left(\epsilon\left(\mathcal{K}^{+}\right), \mathcal{K}^{+}\right),
$$

by modifying the proof of Corollary 2.3. We can then adapt the proofs of Proposition 2.5 and Theorem 2.9 to show that

$$
\beta\left(r_{D}, g\right)=\left|\left\{P \in \mathcal{S} y l \mid P \cap P^{g}=D\right\}\right| 1_{F} .
$$

The number of Sylow 2-subgroups of $G$ which contain $D$ is odd, by a well known generalization of Sylow's Theorem. It then follows from our hypothesis, and $(2.11)$, that $\beta\left(r_{D}, g\right)=1_{F}$. So $G$ has a real 2-block $B \leftrightarrow e \leftrightarrow \omega$ which has defect group $D$, and $\beta\left(e, \mathcal{K}^{+}\right)=\beta(e, g) \neq 0_{F}$. Also $\omega\left(\mathcal{K}^{+}\right)=\omega\left(\mathcal{K}^{o+}\right) \neq 0_{F}$, by Lemma 2.1. This completes the proof. 


\section{Real 2-REgular Classes AND 2-Blocks}

In this section we prove Theorem 3.1 and give a number of applications.

Proof of Theorem 3.1. Recall that $g$ is a 2-regular element of $G$ with defect pair $(D, E)$. Note that if $E \leq P$, then $P g P=P g^{-1} P$.

We claim that $E$ acts on the set $\phi(g):=\left\{P \in \mathcal{S} y l \mid P \cap P^{g}=D, P g P=\right.$ $\left.P g^{-1} P\right\}$ by conjugation. For, suppose that $P \in \phi(g)$. Then $D$ normalizes $P$. If $e \in E \backslash D$ then $g^{e}=g^{-1}$. So $P^{e} g P^{e}=\left(P g^{-1} P\right)^{e}=(P g P)^{e}=P^{e} g^{-1} P^{e}$. Moreover $e g=g^{-1} e$ normalizes $D$. Thus $P^{e} \cap P^{e g}=\left(P^{g} \cap P\right)^{g^{-1} e}=D^{g^{-1} e}=D$. This shows that $P^{e} \in \phi(g)$, which proves our claim.

Each $E$-orbit on $\phi(g)$ has cardinality 1 or 2 . Since $P$ is a Sylow 2-subgroup of $G$, it is stablized by $E$ if and only if $E \leq P$. We conclude that

$$
|\phi(g)| \equiv\left|\left\{P \in \mathcal{S} y l \mid P \cap P^{g}=D, E \leq P\right\}\right|(\bmod 2) .
$$

The result now follows from Theorem 2.9.

In our first application of Theorem 3.1, we give another proof of $[4,4.8]$.

Theorem 3.2. Let $D$ be a 2-subgroup of $G$. Suppose that no subgroup of $\mathbf{N}(D) / D$ is isomorphic to a dihedral group of order 8 . Then $\beta\left(e_{D}, g\right)=1_{F}$, for each real 2-regular element $g$ of $G$ which has defect group D. In particular, the following are equivalent:

(a). G has a real 2-regular element with defect group D;

(b). G has a 2-block with defect group D;

(c). G has a real 2-block with defect group $D$.

Proof. The implications $(\mathrm{c}) \Longrightarrow(\mathrm{b}) \Longrightarrow($ a) follow as in $[4,4.8]$. 
Suppose that $D$ is a Sylow 2-subgroup of $G$. Then the principal 2-block $B_{0} \leftrightarrow e_{0} \leftrightarrow \omega_{0}$ is the only real block with defect group $D$, and the identity class is the only real 2-regular class with defect group $D$. Also $\beta\left(e, 1^{+}\right)=\beta\left(e^{o}, 1^{+}\right)$, for each non-real 2-block idempotent. It follows that $\beta\left(e_{D}, 1^{+}\right)=\beta\left(e_{0}, 1^{+}\right)=$ $\omega_{0}\left(1^{+}\right)=1_{F}$, using Lemma 2.1 (this also follows from a theorem of R. Brauer).

Suppose that $D$ is not a Sylow 2-subgroup of $G$. Let $g$ be a real 2-regular element with defect pair $(D, E)$. The first statement and the implication (a) $\Longrightarrow$ (c) will follow from Theorem 3.1, if we can show that $P \cap P^{g}=D$, whenever $P$ is a Sylow 2-subgroup of $G$ which contains $E$.

Assume for the sake of contradiction that there exists $P \in \mathcal{S} y l$ with $E \leq P$ and $P \cap P^{g}>D$. Let $x \in E \backslash D$, and set $y:=x^{-1} g=g^{-1} x^{-1}$. Then $y \in \mathbf{N}(D)$, since $x \in E \leq \mathbf{N}(D)$ and $g \in \mathbf{C}(D)$. Also $y \in \mathbf{N}\left(P \cap P^{g}\right)$, since $\left(P \cap P^{g}\right)^{y}=$ $P^{x^{-1} g} \cap P^{x^{-1}}=P^{g} \cap P$.

The 2-group $\langle y\rangle$ acts on the nontrivial 2-group $\mathbf{N}_{P \cap P^{g}}(D) / D$. So we can choose $n \in \mathbf{N}_{P \cap P^{g}}(D) \backslash D$ such that $n^{2} \in D$ and $[n, y] \in D$. Our hypothesis on $\mathbf{N}(D) / D$ forces $[n, x] \in D$. Therefore $[n, g]=[n, x y]=[n, x][n, y]^{x^{-1}}$ lies in $D$. It follows that $g$ centralizes $\langle D, n\rangle$, since $\langle g\rangle$ is a 2'-group which acts trivially on every factor of $1 \leq D<\langle D, n\rangle$. This contradicts the fact that $D$ is a defect group of $g$. The theorem follows.

In our next application we give self contained proofs of a number of results on extremal 2-blocks which are due to M. Herzog [6]. We call $G$ a $C I$-group if every intersection of distinct Sylow 2-subgroups of $G$ is centralized by some Sylow 2-subgroup of $G$. It is straightforward to show that every subgroup and factor group of a CI-group is a CI-group. Let $S$ and $T$ be Sylow 2-subgroups of $G$. We say that $S \cap T$ is a maximal Sylow intersection in $G$ if $S \neq T$ and 
whenever $S \cap T \leq P \cap Q$, where $P \neq Q$ are Sylow 2-subgroups of $G$, then $S \cap T=P \cap Q$.

Lemma 3.3. Let $G$ be a CI-group. Suppose that $S \neq T$ are Sylow 2-subgroups of $G$. Then $S \cap T$ is centralized by every 2-group which contains it.

Proof. Let $R$ be a 2-subgroup of $G$ which contains $S \cap T$. Since $S \neq T$, we may assume that $R \neq T$. Then $R \cap T \geq S \cap T$. It is no loss to assume that $R=S$ and moreover that $S \cap T$ is a maximal Sylow intersection in $G$.

Now $\mathbf{Z}(S) \leq \mathbf{C}(S \cap T)$. So we can find a Sylow 2-subgroup $X$ of $G$ which centralizes $S \cap T$ and contains $\mathbf{Z}(S)$. Then $S \cap T \leq X$, since $X$ normalizes $S \cap T$. It follows that $S \cap T \leq S \cap X$. If $S=X$ we are done. So assume that $S \neq X$. Then $S \cap T=S \cap X$, as $S \cap T$ is a maximal Sylow intersection. In particular $\mathbf{Z}(S) \leq S \cap T$. But $S \cap T \leq \mathbf{Z}(X)$ and $|\mathbf{Z}(S)|=|\mathbf{Z}(X)|$. So $\mathbf{Z}(S)=S \cap T=\mathbf{Z}(X)$.

Here is our main result:

Theorem 3.4. Let $G$ be a CI-group. Then $\beta\left(e_{D}, g\right)=1_{F}$, for each real 2regular element $g \in G$ which has defect group $D$. In particular, the statements (a), (b) and (c) of Theorem 3.2 are equivalent.

Proof. The implications (c) $\Longrightarrow(b) \Longrightarrow$ (a) follow as in Theorem 4.8 of [4].

Let $g$ be a real 2-regular element of $G$ which has defect pair $(D, E)$. Choose $s \in E \backslash D$ and set $t:=s g$. Then $s$ and $t$ are 2-elements which invert $g$ and $s^{2}=t^{2}$ lies in $D$. Let $S$ be a Sylow 2-subgroup of $G$ which contains $E$. Then $t \in \mathrm{N}\left(S \cap S^{g}\right)$ since $\left(S \cap S^{g}\right)^{t}=S^{s t} \cap S^{s g t}=S^{g} \cap S^{2}=S^{g} \cap S$. So $\left\langle S \cap S^{g}, t\right\rangle$ is a 2-group which contains $S \cap S^{g}$. We deduce from Lemma 3.3 that $t$ centralizes 
$S \cap S^{g}$. Also $s \in S$ also centralizes $S \cap S^{g}$, again using Lemma 3.3. So $S \cap S^{g}$ is a 2-subgroup of $\mathbf{C}(g)$. It follows that $S \cap S^{g}=D$, as $D \leq S \cap S^{g}$ and $D$ is a Sylow 2-subgroup of $\mathbf{C}(g)$. The first statement and the implication (a) $\Longrightarrow$ (c) now follow as in Theorem 3.2.

We can now prove:

Proposition 3.5. Let $G$ be a CI-group and let $D$ be a maximal Sylow intersection in $G$. Then $G$ has a real 2-block with defect group $D$.

Proof. Note that $D$ is the largest normal 2-subgroup of $\mathbf{N}(D)$, and also that it is not a Sylow 2-subgroup of $\mathbf{N}(D)$.

We claim that $\mathbf{N}(D)$ has a nonidentity real 2-regular element. For suppose otherwise. Then $\mathbf{N}(D) / D$ has no nonidentity real 2-regular elements. It follows from the Baer-Suzuki theorem that $\mathbf{N}(D) / D$ has a nontrivial normal 2 -subgroup, which contradicts the first paragraph.

Theorem 3.4 now shows that $\mathbf{N}(D)$ has a real 2-block $b$ with non-maximal defect. But $b$ has a defect group which contains $D$, by a theorem of $\mathrm{R}$. Brauer. It follows that $D$ is a defect group of $b$. The proposition now follows from Brauer's first main theorem.

Theorems 1 and 2 of [6] are consequences of the following corollaries:

Corollary 3.6. Let $G$ be a finite group. Then $G$ has a normal Sylow 2subgroup if and only if $G$ is a CI-group with no real non-principal 2-blocks.

Proof. The 'only if' part is straightforward. 
Suppose that $G$ is a CI-group which has no real non-principal 2-blocks. Proposition 3.5 implies that $G$ has no maximal Sylow intersections. So $G$ has a normal Sylow 2-subgroup.

We call $G$ a TI-group if every pair of distinct Sylow 2-subgroups of $G$ intersect in the identity.

Corollary 3.7. Let $G$ be a finite group. Then $G$ is a TI-group if and only if $G$ is a CI-group and all real non-principal 2-blocks of $G$ have defect 0 .

Proof. The 'only if' part is straightforward.

Suppose that $G$ is a CI-group and all real non-principal 2-blocks of $G$ have defect 0. We may assume that $G$ does not have a normal Sylow 2-subgroup. Let $D$ be a maximal Sylow intersection in $G$. Then $G$ has a real 2-block with defect group $D$, by Proposition 3.5. It follows from the hypothesis that $D=\{1\}$. So $G$ is a TI-group.

\section{EXtended DEFECT GROUPS FOR REAL 2-Blocks}

In this section we introduce the notion of defect pairs for real 2-blocks. We defined the relation $\preceq$ in (1.1). Now $\preceq$ is almost a partial order, in the sense that if $\mathcal{K}$ and $\mathcal{L}$ are properly real classes and if $\mathcal{M}$ is a real class, then

$$
\mathcal{K} \preceq \mathcal{L} \text { and } \mathcal{L} \preceq \mathcal{M} \quad \Longrightarrow \quad \mathcal{K} \preceq \mathcal{M}
$$

Also

(4.1) $\mathcal{K} \preceq \mathcal{L}$ and $\mathcal{L} \preceq \mathcal{K} \Longrightarrow \mathcal{K}$ and $\mathcal{L}$ have the same defect pairs. 
Set $[\mathcal{K}]:=\mathcal{K} \cup \mathcal{K}^{o}$, for each class $\mathcal{K}$ of $G$, and let

$$
Z^{*}:=\sum F[\mathcal{K}]^{+}
$$

where $\mathcal{K}$ ranges over the classes of $G$. Then $Z^{*}$ is a subalgebra of $Z$, as it coincides with the set of fixed points of the involutary automorphism $x \rightarrow x^{o}$ of $Z$. Each real 2-block idempotent of $F G$ lies in $Z^{*}$. By inspecting the proof of Theorem 2.1 of [3], we see that the following is true:

Proposition 4.2. Suppose that $\mathcal{L}$ is a real class of $G$ and that $\mathcal{K}^{+}$is a properly real class which lies in the ideal of $Z^{*}$ generated by $\mathcal{L}^{+}$. Then $\mathcal{K} \preceq \mathcal{L}$.

R. Gow showed in $[3,1.2]$ that if $B \leftrightarrow e \leftrightarrow \omega$ is a real 2-block of $G$, then there exists a real 2-regular class $\mathcal{K}$ of $G$ such that $\beta\left(e, \mathcal{K}^{+}\right) \neq 0$ and $\omega\left(\mathcal{K}^{+}\right) \neq 0$. He called any such class a real defect class for $B$. We will call the defect pairs of the real defect classes of $B$ the defect pairs of $B$.

Proof of Theorem 4.3. Suppose that $\omega\left(\mathcal{K}^{+}\right) \neq 0$. Then $e=\omega\left(\mathcal{K}^{+}\right)^{-1} \epsilon\left(\mathcal{K}^{+}\right) e$. Also $\epsilon\left(\mathcal{K}^{+}\right)=\left(\mathcal{K}^{+}\right)^{2^{n}}$, for some integer $n>0$, as in Section 2. So $e$ lies in the ideal of $Z^{*}$ which is generated by $\mathcal{K}^{+}$. But $\beta\left(e, \mathcal{L}^{+}\right) \neq 0$. So $\mathcal{L} \preceq \mathcal{K}$, by Proposition 4.2.

Suppose that $\beta\left(e, \mathcal{K}^{+}\right) \neq 0$. Then, using the fact that $\omega\left(\mathcal{L}^{+}\right) \neq 0$, the argument of the previous paragraph shows that $\mathcal{K} \preceq \mathcal{L}$.

Let $P, Q, R$ and $S$ be subgroups of $G$. We say that the pairs $(P, Q)$ and $(R, S)$ are conjugate in $G$ if there exists $g \in G$ such that $R=P^{g}$ and $S=Q^{g}$. Our corollary is an immediate consequence of (4.1) and Theorem 4.3:

Corollary 4.4. The defect pairs of a real 2-block are conjugate in $G$. 


\section{The Number of ReAl 2-Blocks With A GIVEN DEFeCt PAIR}

We begin this section with a result which associates a certain $S$-orbit of 2-groups to each self-dual $(S, S)$-double coset.

Lemma 5.1. Suppose that $x \in G \backslash S$ and that $S x S=S x^{-1} S$. Set $\left(S \cap S^{g}\right)^{*}:=$ $\left(S \cap S^{g}\right) \cup\left(S g \cap g^{-1} S\right)$, for each $g \in S x S$. Then $\left(S \cap S^{g}\right)^{*}$ is a 2-subgroup of $G$ which contains $S \cap S^{g}$ as a subgroup of index 2. Moroever the $\left(S \cap S^{g}\right)^{*}$ forms a single $S$-conjugation orbit, and $S g \cap g^{-1} S$ coincides with the set $\{y \in$ $\left.S g \mid y^{2} \in S \cap S^{g}\right\}$.

Proof. First we show that $S g \cap g^{-1} S$ is nonempty. We may write $g^{-1}=s g t$, for certain $s, t \in S$. Then $s g=g^{-1} t$ is an element of $S g \cap g^{-1} S$.

We claim that $S g \cap g^{-1} S$ is a right $\left(S \cap S^{g}\right)$-coset. Let $a=b^{g} \in S \cap S^{g}$, where $a, b \in S$, and let $c g=g^{-1} d \in S g \cap g^{-1} S$, where $c, d \in S$. Then $(c g) a$ also lies in $S g \cap g^{-1} S$ since $g^{-1}(d a)=(c g) a=(c b) g$. Also $(c g)(s g)^{-1}$ lies in $S \cap S^{g}$ since $c s^{-1}=(c g)\left(g^{-1} s^{-1}\right)=\left(g^{-1} d\right)\left(t^{-1} g\right)=\left(d t^{-1}\right)^{g}$. This proves our claim.

Now $s g \in \mathbf{N}\left(S \cap S^{g}\right)$, since $\left(S \cap S^{g}\right)^{s g}=S^{s g} \cap S^{t}=S^{g} \cap S$. It follows from this and the previous paragraphs that $\left(S \cap S^{g}\right)^{*}$ is a subgroup of $G$, which contains $S \cap S^{g}$ as a subgroup of index 2 .

Write $g=u x v$, where $u, v \in S$. Then it is clear that $\left(S \cap S^{g}\right)^{*}=\left(S \cap S^{x}\right)^{* v}$. So the 2-groups $\left(S \cap S^{g}\right)$ * form a single $S$-orbit of subgroups of $G$.

Finally, suppose that $y=z g$, for $z \in S$. If $y^{2} \in S \cap S^{g}$, then $y^{2}=u^{g}$, for some $u \in S$. Thus $y=\left(g^{-1} u g\right)\left(g^{-1} z\right)=g^{-1} u z$ lies in $S g \cap g^{-1} S$. Conversely, suppose that $y \in S g \cap g^{-1} S$. Then $y=g^{-1} v$, for some $v \in S$. Hence $z v=y^{2}=(v z)^{g}$ lies in $S \cap S^{g}$. This proves the last statement of the lemma. 
The subgroups $\left(S \cap S^{g}\right)^{*}$ have appeared in the literature on self-inverse double cosets. See for example 12.13.(ii) of [2].

Let $\mathcal{K}$ and $\mathcal{L}$ be 2-regular conjugacy classes of $G$, which have defect groups $D$ and $Q$ respectively. Let $x$ be any element of $G$. The 2-group $S \cap S^{x}$ acts by conjugation on $\mathcal{K} \cap S x$ and on $\mathcal{L} \cap S x$. So $|\mathcal{K} \cap S x| \equiv|\mathbf{C}(\mathcal{K}, x)|(\bmod 2)$, where $\mathbf{C}(\mathcal{K}, x):=\mathcal{K} \cap S x \cap \mathbf{C}\left(S \cap S^{x}\right)$. Let $\mathbf{O}(\mathcal{L}, x)$ denote the set of those orbits of $S \cap S^{x}$ on $\mathcal{L} \cap S x$ which have a representative $l$ such that $S \cap S^{x}$ contains a Sylow 2-subgroup of $\mathbf{C}(l)$. Now $|\mathcal{L} \cap S x|=\sum\left|S \cap S^{x}: \mathbf{C}_{S \cap S^{x}}(l)\right|$. So $\left(|Q||\mathcal{L} \cap S x| /\left|S \cap S^{x}\right|\right)$ is an integer which has the same parity as $|\mathbf{O}(\mathcal{L}, x)|$.

We will use $\sum_{x}^{Q<D}$ denote a sum which ranges over those double cosets $S x S$ for which $Q \leq_{G} S \cap S^{x} \leq_{G} D$, and $\sum_{x \equiv x^{-1}}^{Q<D}$ to denote the restriction of this sum to the self-dual double cosets. These sums are empty unless $Q \leq_{G} D$. It follows from $[12,1.3 .3$ and 1.3.4], and the fact that $\operatorname{Char}(F)=2$, that

$$
\beta\left(\epsilon\left(\mathcal{K}^{+}\right), \mathcal{L}^{+}\right)=\sum_{x}^{Q<D}|\mathbf{C}(\mathcal{K}, x)||\mathbf{O}(\mathcal{L}, x)| 1_{F} .
$$

Let $\mathbf{C}^{*}(\mathcal{K}, x)$ denote the set of elements of $\mathbf{C}(\mathcal{K}, x)$ which are inverted by some element of $\left(S \cap S^{x}\right)^{*}$, and let $\mathbf{O}^{*}(\mathcal{L}, x)$ denote the set of orbits in $\mathbf{O}(\mathcal{L}, x)$ whose elements are inverted by some element of $\left(S \cap S^{x}\right)^{*}$.

Proposition 5.3. Suppose that $\mathcal{K}$ and $\mathcal{L}$ are real 2 -regular classes of $G$, with defect groups $D$ and $Q$ respectively. Then

$$
\beta\left(\epsilon\left(\mathcal{K}^{+}\right), \mathcal{L}^{+}\right)=\sum_{x \equiv x^{-1}}^{Q<D}\left|\mathbf{C}^{*}(\mathcal{K}, x)\right|\left|\mathbf{O}^{*}(\mathcal{L}, x)\right| 1_{F} .
$$


Proof. By pairing each double coset in (5.2) with its dual, as in the proof of Proposition 2.5, we see that

$$
\beta\left(\epsilon\left(\mathcal{K}^{+}\right), \mathcal{L}^{+}\right)=\sum_{x \equiv x^{-1}}^{Q<D}|\mathbf{C}(\mathcal{K}, x)||\mathbf{O}(\mathcal{L}, x)| 1_{F} .
$$

Suppose that $S x S=S x^{-1} S$, where $x \in G$. Let $s x \in S x$ and $t x=x^{-1} u \in$ $S x \cap x^{-1} S$, where $s, t, u \in S$. Then $(s x)^{-t x}=\left(u^{-1} x\right)\left(x^{-1} s^{-1}\right)(t x)=u^{-1} s^{-1} t x$ also lies in $S x$. Set

$$
y \cdot z:=\left\{\begin{array}{cl}
y^{z}, & \text { if } z \in S \cap S^{x} \\
\left(y^{-1}\right)^{z}, & \text { if } z \in\left(S \cap S^{x}\right)^{*} \backslash\left(S \cap S^{x}\right) .
\end{array}\right.
$$

for each $y \in S x$ and $z \in\left(S \cap S^{x}\right)^{*}$. It is straightforward to show that this defines an action of the 2-group $\left(S \cap S^{x}\right)^{*}$ on $S x$.

Now $\left(S \cap S^{x}\right)^{*}$ stabilizes $\mathbf{C}(\mathcal{K}, x)$, and also each $S \cap S^{x}$-orbit in $\mathbf{O}(\mathcal{L}, x)$. Since $\left(S \cap S^{x}\right)^{*}$ is a 2-group, this implies that

$$
|\mathbf{C}(\mathcal{K}, x)| \equiv\left|\mathbf{C}^{*}(\mathcal{K}, x)\right|(\bmod 2) \quad \text { and } \quad|\mathbf{O}(\mathcal{L}, x)| \equiv\left|\mathbf{O}^{*}(\mathcal{L}, x)\right|(\bmod 2) .
$$

The proposition follows from this.

Proof of Theorem 5.2. Recall the notation established in Section 1.

Let $B_{1} \leftrightarrow e_{1} \leftrightarrow \omega_{1}, \ldots, B_{u} \leftrightarrow e_{u} \leftrightarrow \omega_{u}$, be a complete list of the (real) 2 -blocks of $G$ which have defect pair $(D, E)$. Suppose that $1 \leq i, j \leq u$. Then

$$
\delta_{i j}=\omega_{j}\left(e_{i}\right)=\sum \beta\left(e_{i}, \mathcal{K}^{+}\right) \omega_{j}\left(\mathcal{K}^{+}\right)
$$

where $\mathcal{K}$ runs through the conjugacy classes of $G$. Suppose that $\mathcal{K} \neq \mathcal{K}^{o}$. Then the contribution of $\mathcal{K}$ and $\mathcal{K}^{o}$ to (5.5) is

$$
\beta\left(e_{j}, \mathcal{K}^{+}\right) \omega_{i}\left(\mathcal{K}^{+}\right)+\beta\left(e_{j}, \mathcal{K}^{o+}\right) \omega_{i}\left(\mathcal{K}^{o+}\right)=2 \cdot \beta\left(e_{j}, \mathcal{K}^{o+}\right) \omega_{i}\left(\mathcal{K}^{o+}\right)=0 .
$$


Also any real class which occurs with non-zero multiplicity in (5.5) is 2-regular and is not the trivial class. So any such class is properly real. It follows from Theorem 4.3 that

$$
\delta_{i j}=\sum_{k=1}^{v} \beta\left(e_{i}, \mathcal{K}_{k}^{+}\right) \omega_{j}\left(\mathcal{K}_{k}^{+}\right) .
$$

Form the $u \times v$-matrices $A$ and $B$ by setting the $i, j$-th entry of $A$ as $A_{i j}=$ $\beta\left(e_{i}, \mathcal{K}_{j}^{+}\right)$and the $i, j$-th entry of $B$ as $B_{i j}=\omega_{i}\left(\mathcal{K}_{j}^{+}\right)$. Then $A B^{T}$ is the $u \times u$ identity matrix, by (5.6). It follows that the $v \times v$-matrix $B^{T} A$ has rank $u$.

Suppose that $B \leftrightarrow e \leftrightarrow \omega$ is a non-real 2-block of $G$ and that $1 \leq i, j \leq$ $v$. Then, since $\mathcal{K}_{i}=\mathcal{K}_{i}^{o}$ and $\mathcal{K}_{j}=\mathcal{K}_{j}^{o}$, the contribution of $e$ and $e^{o}$ to $\beta\left(\epsilon\left(\mathcal{K}_{i}^{+}\right), \mathcal{K}_{j}^{+}\right)$is

$$
\omega\left(\mathcal{K}_{i}^{+}\right) \beta\left(e, \mathcal{K}_{j}^{+}\right)+\omega^{o}\left(\mathcal{K}_{i}^{+}\right) \beta\left(e^{o}, \mathcal{K}_{j}^{+}\right)=2 \cdot \omega\left(\mathcal{K}_{i}^{+}\right) \beta\left(e, \mathcal{K}_{j}^{+}\right)=0 .
$$

Thus

$$
\beta\left(\epsilon\left(\mathcal{K}_{i}^{+}\right), \mathcal{K}_{j}^{+}\right)=\beta\left(\sum \omega\left(\mathcal{K}_{i}^{+}\right) e, \mathcal{K}_{j}^{+}\right)=\sum \omega\left(\mathcal{K}_{i}^{+}\right) \beta\left(e, \mathcal{K}_{j}^{+}\right),
$$

where $B \leftrightarrow e \leftrightarrow \omega$ runs through the real 2-blocks of $G$. So by Theorem 4.3 we have

$$
\beta\left(\epsilon\left(\mathcal{K}_{i}^{+}\right), \mathcal{K}_{j}^{+}\right)=\sum_{k=1}^{u} \omega_{k}\left(\mathcal{K}_{i}^{+}\right) \beta\left(e_{k}, \mathcal{K}_{j}^{+}\right) .
$$

The sum on the right hand side is the $i, j$-th entry of the matrix $B^{T} A$. We conclude that the $v \times v$ matrix $M$ with $i, j$-th entry $M_{i j}=\beta\left(\epsilon\left(\mathcal{K}_{i}^{+}\right), \mathcal{K}_{j}^{+}\right)$has rank $u$.

It now follows from Proposition 5.3 that $u=0$ if $w=0$, and

$$
M_{i, j}=\sum_{k=0}^{w} \mathbf{C}^{*}\left(\mathcal{K}_{i}, x_{k}\right) \mathbf{C}^{*}\left(\mathcal{K}_{j}, x_{k}\right),
$$


if $w>0$. But $\mathbf{C}^{*}\left(\mathcal{K}_{i}, x_{k}\right)=N_{i k}$ and $\mathbf{C}^{*}\left(\mathcal{K}_{j}, x_{k}\right)=N_{j k}$. We conclude that $M=N \cdot N^{T}$, which completes the proof.

\section{REFERENCES}

[1] R. Brauer, On Blocks and Sections in Finite Groups I, Amer. J. Math. 89 (4) (1967), $1115-1136$.

[2] C. Curtis, I. Reiner, Methods of Representation Theory, I, John Wiley, New York, 1981.

[3] R. Gow, Real 2-blocks of characters of finite groups, Osaka J. Math. 25 (1988), 135-147.

[4] R. Gow, J. Murray, Real 2-regular classes and 2-blocks, to appear, J. Algebra.

[5] J. A. Green, Blocks of modular representations, Math. Zeit. 79 (1962), 100-115.

[6] M. Herzog, On groups with extremal blocks, Bull. Austral. Math. Soc. 14 (1976), 325330.

[7] I. M. Isaacs, Character Theory of Finite Groups, Dover Publ., Inc., New York (1994).

[8] J. Murray, Blocks of defect zero and products of elements of order p, J. Algebra 214 (1999), 385-399.

[9] M. F. O'Reilly, On a Theorem of J. A. Green, J. Austral. Math. Soc. 20 (Series A) (1975), 449-450.

[10] M. Osima, On block idempotents of modular group rings, Nagoya Math. J. 27 (1966), 429-433.

[11] G. R. Robinson, The Number of Blocks with a Given Defect Group, J. Algebra 84 (1983), 493-502.

[12] G. R. Robinson, Double Cosets and Modular Representation Theory, Proc. Symp. Pure Math. 47 (1987), 249-258. 